\title{
The Role of Social Support and the Neighborhood Environment on Physical Activity in Low-income, Mexican-American Women in South Texas
}

\author{
Jennifer J. Salinas', Marisol McDaniel' ${ }^{2}$, Deborah Parra-Medina ${ }^{2}$ \\ ${ }^{1}$ Center of Emphasis in Cancer, Department of Biomedical Sciences, Texas Tech University Health Sciences Center EI Paso, EI Paso, TX, USA; \\ ${ }^{2}$ Latino Research Initiative, College of Liberal Arts, University of Texas at Austin, Austin, TX, USA
}

Objectives: To determine the relationships between physical activity (PA), the neighborhood environment support for PA, and social support for PA among Mexican-American women living in South Texas. The Enlace study was a randomized controlled trial that tested the effectiveness of a promotora-led PA intervention among low-income Mexican origin women $(n=614)$ living in colonias.

Methods: The dependent measures included accelerometer-measured average moderate to vigorous physical activity (MVPA) and sedentary breaks and the Community Health Activities Model Program for Seniors PA 41-item questionnaire. The independent measures included the Physical Activity and Neighborhood Environment Scale (PANES) and the 13-item Physical Activity Social Support (PASS) scale.

Results: Enlace participants were on average 40.4 (standard deviation, 10.3) years old, born in Mexico (86.1\%), and uninsured (83.1\%). Adjusted linear regression results indicated that each 1-point increment in the PANES overall score was associated with 0.050 $(p<0.001)$ unit increase in sedentary break and a $-0.043(p=0.001)$ unit decrease in sedentary break duration. Both PANES $(\beta=0.296$; $p=0.002)$ and PASS scores $(\beta=0.076 ; p<0.001)$ were associated with weekly average self-reported MVPA. Interaction effects were observed between PASS scores and accelerometer-measured frequency of sedentary breaks and sedentary time duration.

Conclusions: The findings of this study indicate that the relationships between PA and built environment and social support are measure-dependent and suggest that reducing sedentary time in this population may require a closer assessment of social support for PA.

Key words: Environmental design, Health equity, Community-based participatory research, Exercise

\section{INTRODUCTION}

The Texas-US-Mexico border is one of the most economically challenged areas of the country [1]. Mexican-Americans in

Received: February 27, 2018 Accepted: July 17, 2018

Corresponding author: Marisol McDaniel, DrPH

Latino Research Initiative, College of Liberal Arts, University of Texas at Austin, 210 W. 24th Street, Austin, TX 78712, USA

E-mail: marisol.d.mcdanie|@austin.utexas.edu

This is an Open Access article distributed under the terms of the Creative Commons Attribution Non-Commercial License (http://creativecommons.org/licenses/by$\mathrm{nc} / 4.0 /$ ) which permits unrestricted non-commercial use, distribution, and reproduction in any medium, provided the original work is properly cited. this region are more likely to be uninsured, live in extreme poverty, and have higher disease burdens than non-border Mexican-Americans in Texas and other groups nationally [2]. Individuals living in extreme poverty do not have the resources to freely engage in physical activity (PA), such as gym memberships, or class fees that may facilitate activities, as do the more affluent [3]. As a result, according to the Behavioral Risk Factor Surveillance System, physical inactivity in this region is higher than in Texas and the US (36.8 vs. 26.7 vs. $24.4 \%$ ) [4].

In the current study, we analyzed a unique sample of Mexican-American women residing in South Texas to determine the relationships between $\mathrm{PA}$, neighborhood environment, 
and social support for PA. The Enlace study was a randomized controlled trial study that tested the effectiveness of a promotora-led PA intervention with Mexican-origin women living in unincorporated settlements (colonias) that often lack basic infrastructure, such as indoor plumbing, running water, and electricity [2]. In colonias, streets are usually unpaved, and there is limited greenspace for PA for women residing there.

Neighborhood environment is a strong and consistent predictor of PA $[5,6]$. Amenities such as parks, walking trails, and sidewalks increase PA $[7,8]$. In addition, neighborhood aesthetics such as the presence of trees, abandoned buildings or homes, graffiti, violence, and crime have all been documented as predictors of level of activity among residents $[8,9]$. However, these relationships are complex, and there is substantial variation among metropolitan areas, populations with different distributions of ethnicities, and rural/urban settings $[8,10]$. Additionally, much of what is known about neighborhood built environment and PA is drawn from urban settings or multiethnic samples [8-10], and there is limited information on ruralresiding Hispanics, a population at increased risk for PA [11].

There is substantial evidence that social support is an aspect of Hispanic culture that is protective of health $[12,13]$. Numerous articles have shown that Hispanics who receive or perceive greater levels of social support report lower levels of depression, stress, and chronic diseases [12,14-16]. Evidence also exists that more supportive social environments may contribute to better mental wellbeing and increased PA engagement among women $[9,12]$. Women who engage in PA through group activities or have greater social support to be active are more likely to consistently incorporate PA into their routines [17]. Social support may be one way that Hispanic women are able to offset the effects of suboptimal neighborhood environment for PA, like colonias in South Texas and other impoverished regions of the US. However, this potential relationship has not been fully explored.

Due to the uniqueness of the sample-very-low-income Mexican-origin women living in the Texas-Mexico border region with multiple subjective and objective measures of PAthis study provides important insights into how neighborhood built environment, social support, and PA are associated in an understudied subpopulation of Hispanics. This study also addresses the dearth of knowledge on the relationship between neighborhood built environment and social support for PA in Hispanic subpopulations. Having social support for healthy behaviors increases the likelihood of engaging in salubrious activities [18]. Neighbors often serve as sources of moral support for physical and mental health changes and neighborhood social capital has a significant impact on health and wellbeing $[19,20]$. In neighborhoods where the built environment does not support PA, social support and encouragement from family and neighbors is essential to counter the negative effects of limited resources to be active. We set out to determine to what extent perceived support for PA in the built environment was associated with actual objective and subjective measures of PA. We assessed how social support and built environment were individually associated with varying measures of PA. Additionally, we investigated whether the relationship between neighborhood built environment and PA was attenuated by social support as a potential buffer against the negative effects of poverty.

\section{METHODS}

\section{Participants}

Enlace was a promotora-led PA intervention in the Rio Grande Valley of South Texas on the Texas-Mexico border. The 614 Hispanic women (age, 18-64 years) who participated in this study were recruited from unincorporated, rural housing settlements known as colonias [2]. Participants who did not meet recommended PA guidelines participated in a 16-week long intervention that included education, demonstrations, walking groups, and group-based exercise classes. Baseline PA and perceived neighborhood quality were used for this analysis.

\section{Variables}

\section{Dependent variables}

Objective measures

We utilized multiple measures collected using an accelerometer. Previous studies have traditionally used moderate to vigorous physical activity (MVPA) as the gold standard of activity measurement [21]. However, many are unable to meet that level, therefore, focusing only on MVPA oversimplifies PA outcomes to a level achievable by few. We assert that to fully appreciate the complexity of PA as it pertains to social support and neighborhood built environment, it was important to consider both the traditional MVPA measure and alternatives, such as sedentary time.

Participants wore an accelerometer over 7 consecutive days. The Actigraph GT3X $16 \mathrm{Mb}$ activity monitor is small, lightweight, and designed to detect vertical acceleration, which al- 
lows for the detection of normal human motion and does not register high-frequency vibrations encountered in activities such as operating a lawnmower. The filtered acceleration signal is digitized, and the magnitude is summed over a userspecified time interval. The total minutes per week at baseline were recorded, as well as intensity. For this study, we report the average daily MVPA, the average number of sedentary breaks, the duration of time of each sedentary break, and total daily sedentary time. Intensity was measured using cut points established by Kozey Keadle et al. [22]. We distinguished between the duration of time of sedentary breaks and the total number of breaks due to a growing body of literature distinguishing the health effects of the frequency of breaks from those of the overall time spent in sedentary activity [23-25]. To adjust for significant skewness, we performed natural logarithm on each of these outcome variables.

\section{Subjective measures}

The Community Health Activities Model Program for Seniors (CHAMPS) PA questionnaire is a 41-item self-report measure of PA that was developed originally for older adults, but has been used with the general adult population. We modified age-specific items to be more age-neutral (e.g., replacing "visit a senior center" with "visit a community center"). Responses were used to calculate multiple measures of PA. For this study, we made use of the calculated weekly average MVPA, measured with the Actigraph monitor, and took advantage of walking variables within the CHAMPS index as a common measure of PA, particularly for those with limited access to economic resources associated with other forms of PA. Three scale items assessed the levels of brisk walking, errand walking, and leisure walking. We used hours per week as an outcome measure, categorized as less than 2, 2-3, and more than 3 hours.

\section{Independent variables}

The Physical Activity and Neighborhood Environment Scale (PANES) is an instrument used to assess neighborhood attributes as they relate to aesthetics and walkability. Responses were based on Likert-scale response options, with a higher score representing greater neighborhood environmental support for PA.

The Physical Activity Social Support (PASS) is a 13-item scale developed by Sallis et al. [26] that measures family and friend support of PA. This scale has been tested for reliability and validity $[26,27]$.

\section{Covariates}

Covariates included age (continuous), years of education (continuous), monthly household income, country of birth (US, Mexico, other), health insurance (yes/no), and percentage body fat, which was used as a more precise measure of obesity than body mass index.

\section{Analysis}

Descriptive statistics on the socio-demographic characteristics of the study sample were first conducted to identify potential confounders in the data. A distribution analysis was then conducted on both the primary explanatory variables and all PA outcome variables. To account for skewness and non-normal distributions in the data, natural logarithm were performed on the accelerometer data, and walking variables were categorized into 3 categories. Regression analysis was conducted to test the associations between the neighborhood built environment (PANES) scores, PASS scores, and objective and subjective PA measures, adjusting for significant covariates. Adjusted interaction models were then tested to determine the attenuating effect of social support for PA on the relationship between the neighborhood built environment and the PA outcomes.

\section{RESULTS}

Enlace participants on average had completed 9.9 years (standard deviation [SD], 4.7 years) of education, were 40.4 years old (SD, 10.3 years), and reported a monthly household income of $\$ 1098.3$ (SD, \$1036.0) (Table 1). The majority of participants were born in Mexico (86.1\%) and had no insurance (83.1\%). Finally, the average percentage body fat composition was $39.9 \%$, close to the threshold of $40.0 \%$ that has been documented as being the equivalent of the obese range of body mass index.

Participants tended to evaluate their neighborhood built environment (mean, 2.3; range, 0-6; with 6 being more favorable) and social support for PA (mean, 35.3; range, 16-64; with 64 being the highest level of social support) unfavorably (Table 2). On average, participants reported $3 \mathrm{hr} / \mathrm{wk}$ of MVPA (SD, 4.1). The majority reported less than $2 \mathrm{hr} / \mathrm{wk}$ of brisk walking (81.5\%), errand walking (62.4\%), or leisure walking (62.2\%). A smaller proportion of women reported more than $3 \mathrm{hr} / \mathrm{wk}$ of errand walking (16.6\%), leisure walking (13.4\%), and brisk walking (7.8\%). 
Table 1. Demographic characteristics and body fat measurements of participants in the Enlace cohort

\begin{tabular}{lc}
\hline Characteristics & Total \\
\hline Age (y) & $40.4 \pm 10.3$ \\
Years of education & $9.9 \pm 4.7$ \\
Monthly household income (US dollar) & $1098.3 \pm 1036.0$ \\
Country of birth & \\
United States & $80(12.9)$ \\
Mexico & $533(86.1)$ \\
Other & $6(1.0)$ \\
Health insurance & \\
No & $512(83.1)$ \\
Yes & $104(16.9)$ \\
Body fat (\%) & $39.9 \pm 7.5$
\end{tabular}

Values are presented as number (\%) or mean \pm standard deviation.

Table 2. Baseline demographic characteristics and anthropometrics for the Enlace cohort

\begin{tabular}{lc}
\hline Characteristics & Total \\
\hline Primary explanatory variables & \\
PANES (0-6) & $2.3 \pm 1.7$ \\
PASS (16-64) & $35.3 \pm 9.9$ \\
Physical activity outcome variables & \\
Objective measures (accelerometer) & \\
LN average MVPA per day (0.51-4.63) & $2.8 \pm 0.7$ \\
LN average sedentary breaks (8.30-10.30) & $9.3 \pm 0.4$ \\
LN average sedentary break duration (3.57-6.68) & $5.3 \pm 0.5$ \\
Subjective measures (CHAMPS) & \\
MVPA & $3.0 \pm 4.1$ \\
Walking (hr/wk) & \\
Brisk & \\
$<2$ & $504(81.5)$ \\
$2-3$ & $66(10.7)$ \\
$>3$ & $48(7.8)$ \\
Errand & \\
$<2$ & $386(62.4)$ \\
$2-3$ & $130(21.0)$ \\
$>3$ & $103(16.6)$ \\
Leisure & \\
$<2$ & $385(62.2)$ \\
$2-3$ & $151(24.4)$ \\
$>3$ & $83(13.4)$ \\
\hline
\end{tabular}

Values are presented as mean \pm standard deviation or number (\%).

PANES, Physical Activity and Neighborhood Environment Scale; PASS, Physical Activity Social Support; LN, natural logarithm; MVPA, moderate to vigorous physical activity; CHAMPS, Community Health Activities Model Program for Seniors.

As Table 3 shows, the adjusted linear regression results for accelerometer data indicated that PANES scores were positive-
Table 3. Regression analysis ${ }^{1}$ results for the relationships between PANES, PASS, and objective and subjective measures of physical activity and fitness

\begin{tabular}{|c|c|c|}
\hline & $\begin{array}{l}\text { PANES } \\
\text { (p-value) }\end{array}$ & $\begin{array}{c}\text { PASS } \\
\text { (p-value) }\end{array}$ \\
\hline \multicolumn{3}{|l|}{ Objective measures (accelerometer, per day) } \\
\hline LN average MVPA & $-0.008(0.63)$ & $0.000(0.99)$ \\
\hline LN average sedentary breaks & $0.050(<0.001)$ & $0.003(0.08)$ \\
\hline LN average sedentary break duration & $-0.043(0.001)$ & $-0.004(0.07)$ \\
\hline \multicolumn{3}{|l|}{ Subjective measures (CHAMPS) } \\
\hline MVPA & $0.296(0.002)$ & $0.076(<0.001)$ \\
\hline \multicolumn{3}{|l|}{ Walking (OR, hr/wk) } \\
\hline \multicolumn{3}{|l|}{ Brisk } \\
\hline$<2$ & \multicolumn{2}{|c|}{ Reference } \\
\hline $2-3$ & $1.10(0.21)$ & $1.03(0.05)$ \\
\hline$>3$ & $1.16(0.11)$ & $1.04(0.006)$ \\
\hline \multicolumn{3}{|l|}{ Errand } \\
\hline$<2$ & \multicolumn{2}{|c|}{ Reference } \\
\hline $2-3$ & $1.06(0.39)$ & $1.03(0.006)$ \\
\hline$>3$ & $1.13(0.08)$ & $1.03(0.005)$ \\
\hline \multicolumn{3}{|l|}{ Leisure } \\
\hline$<2$ & \multicolumn{2}{|c|}{ Reference } \\
\hline $2-3$ & $0.98(0.70)$ & $1.03(0.007)$ \\
\hline$>3$ & $1.09(0.23)$ & $1.06(<0.001)$ \\
\hline
\end{tabular}

PANES, Physical Activity and Neighborhood Environment Scale; PASS, Physical Activity Social Support; LN, natural logarithm; MVPA, moderate to vigorous physical activity; CHAMPS, Community Health Activities Model Program for Seniors; OR, odds ratio.

${ }^{1}$ Adjusted for age, grade, household income, country of birth, insurance status, and $\%$ body fat.

ly associated with a $0.050(p<0.001)$ unit increase in the number of sedentary breaks per day and a $-0.043(p=0.001)$ unit decrease in sedentary break duration. There were no significant associations with the accelerometer outcomes or PASS scores. Concerning subjective measures, both PANES $(\beta=0.296$; $p=0.002)$ and PASS scores $(\beta=0.076 ; p<0.001)$ were associated with a higher weekly average time spent engaged in MVPA using the CHAMPS instrument. With each 1-unit increase in the PASS score, there were greater odds of engaging in 2-3 hr/ wk (odds ratio [OR], 1.03; $p=0.049$ ) or more than $3 \mathrm{hr} / \mathrm{wk}$ (OR, $1.04 ; p=0.006$ ) of brisk walking. Similarly, a 1 -unit increase in the PASS score was associated with $1.03(p=0.006)$ greater odds of errand walking for 2-3 hr/wk and $1.03(p=0.005)$ greater odds of errand walking for more than $3 \mathrm{hr} / \mathrm{wk}$ than walking less than $2 \mathrm{hr} / \mathrm{wk}$. Finally, a 1-unit increase in the PASS score was associated with $1.03(p=0.007)$ increased odds of leisure walking for $2-3 \mathrm{hr} / \mathrm{wk}$ and $1.06(p<0.001)$ greater odds 


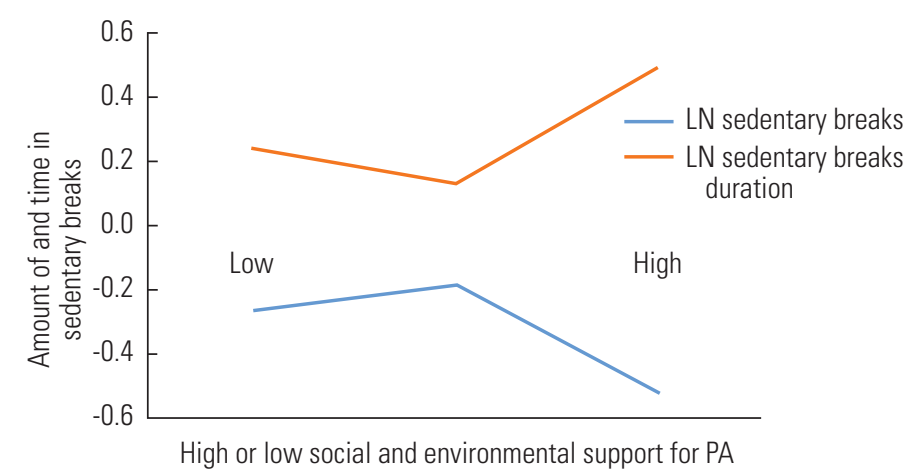

Figure 1. Interaction effects between environmental support and social support for physical activity (PA). LN, natural logarithm.

of leisure walking for more than $3 \mathrm{hr} / \mathrm{wk}$.

Interaction effect models demonstrated a significant interaction effect between PANES and PASS scores for the natural logarithm of sedentary breaks and sedentary break duration (Figure 1). On average, social support for PA reduced the strength of the relationship between the PANES score and natural logarithm of sedentary breaks at higher levels of environmental support for PA. On the contrary, social support for $P A$ increased the strength of the relationship between the PANES and the natural logarithm duration of sedentary breaks. On average, higher social support for PA increased the effect of the PANES score on the length of time participants spent in sedentary breaks $(\beta=0.077 ; p<0.05)$.

\section{DISCUSSION}

Limited information currently exists on PA in very-low-income rural Hispanic communities. The main purpose of this study was to provide a better understanding of how social support, a potential aspect of Hispanic culture that is believed to be protective of health, may offset the negative impact of an unfavorable built environment for PA. The results of our analysis indicate that neighborhood built environment was positively associated with the number of sedentary breaks and subjective MVPA, but negatively associated with sedentary break duration. Additionally, social support was not associated with objective measures of PA, but was positively associated with subjective measures of MVPA, brisk walking, errand walking, and leisure walking using the CHAMPS instrument. Interaction effects demonstrated a significant interaction between neighborhood built environment and social support for the number of sedentary breaks and sedentary break duration only.

Previous work on the relationship between neighborhood built environment and PA have found a more favorable built environment to be associated with higher levels of MVPA [28]. A strength of our study was the inclusion of better-defined measures of PA and sedentary behavior in an understudied Hispanic sub-population. Having done so, we have found that the relationship between PA and built environment may depend on the measure. Neighborhood built environment was significantly associated with increased hours per day of subjective MVPA. Using objective measures from accelerometry, we did not find an association with MVPA; however, a more favorable neighborhood built environment was associated with a higher number of daily sedentary breaks, but shorter durations of sedentary time.

Social support is a mechanism that is often cited as a protective factor from negative health outcomes in Hispanic cultures [29]. In this study, we used social support for PA to test to what extent this potential facet of Hispanic culture may influence PA. Findings from this study revealed that social support for PA was significantly associated with subjective measures, but not objective measures. Women who perceived greater levels of social support for PA reported significantly greater time walking on the CHAMPS instrument. Walking groups have become an increasingly common form of PA because of the potential for socializing [30]. Women in our sample who had greater social support for PA may also have engaged in walking with neighbors, friends, or family, who also may be the main source of social support for PA. Much of the literature related to PA and social support has focused on MVPA or other more vigorous activity endpoints, which are often unachievable in lowincome populations [31]. Since walking may have as much cardiovascular benefit as more intense activities [32], the evidence from this study suggests that finding ways to provide social support to walk may be an effective strategy to encourage low-income women to be active.

Social support for PA significantly attenuated the relationship between built environment and sedentary time. While social support for PA reduced the effect of built environment on the number of sedentary breaks, it increased the effect of the time spent in sedentary breaks. These findings are novel since few studies have examined the relationship between sedentary time and built environment or how social support might moderate this association. These findings again provide insight into the important role of social support in this com- 
munity. A growing body of evidence indicates that shorterduration and more frequent sedentary breaks have important health benefits [23-25]. While social support may be a facilitator of walking, it may inadvertently offset the positive effects of a built environment that is more supportive of a healthy balance between active living and sedentary time. A more favorable built environment may be more conducive to socialization and interaction among neighbors, making residents more inclined to sit outside rather than be active [33]. The few studies that have examined the relationship between built environment and sedentary time have found contradictory associations to ours, in that a more favorable built environment was associated with less sedentary time [34]. However, these studies were conducted in primarily urban settings and with non-Hispanics [35]. Furthermore, previous studies have not looked at how social support might affect this relationship, particularly in economically challenged neighborhoods. In Hispanic neighborhoods that may be conducive to PA, social control or perceived judgment from other residents may serve as a barrier to being active. Promoting social support for PA in these communities may require a change to the PA 'culture' of the neighborhood to overcome potential social barriers [36]. Further, promoting PA that incorporate socialization might be an effective strategy for taking advantage of more favorable built environments in low-income Hispanic communities.

While there are many strengths to this study, some limitations should be pointed out. First, while we see the sample as a strength since it is an understudied subpopulation of a larger Hispanic group, our findings are only applicable to the ruralresiding Hispanic population living along the South Texas, USMexico border. Additionally, the sample was only women, and if we had included men in our analysis, we may have obtained different findings. Additionally, we measured social support for PA using the PASS score, which we believe is another strength of this current study, but using other measures of social support might have yielded different findings. Finally, our analysis was cross-sectional, and therefore we cannot make any causal inferences regarding the effects of built environment or social support on the PA outcomes in this study.

Despite the limitations, there are many notable strengths of this study. First, participants were recruited from a rural, primarily Hispanic community living on the US-Mexico border. The US-Mexico border is a region with an understudied population, for which distinct health outcomes have been documented in comparison to the larger Mexican-American and
Hispanic populations [37]. Additionally, this study made use of both objective and subjective measures of PA, which yielded important findings for both built environment and social support. Further, we included both sedentary time and walking as 2 alternative measures to the standard MVPA. We believe that by including a more diverse range of measures, we were better able to shed light on these relationships in Hispanic and other racial/ethnic groups.

Future research should further examine the relationships between built environment, social support, and alternative measures of PA to develop better-targeted interventions that are more effective for reducing sedentary time and increasing alternative forms of PA that are more achievable in health-disparate groups, such as in this study. Additionally, future studies should examine in greater depth the influence of social support on PA in Hispanics, as doing so may provide greater insight into how social support might serve as a buffer against poorer health outcomes in this population.

\section{ACKNOWLEDGEMENTS}

Research reported in this publication was supported by National Heart, Lung, and Blood Institute (NHLBI) of the National Institutes of Health under award number R01HL111718.

\section{CONFLICT OF INTEREST}

The authors have no conflicts of interest associated with the material presented in this paper.

\section{ORCID}

Jennifer J. Salinas https://orcid.org/0000-0001-6276-7184

Marisol McDaniel http://orcid.org/0000-0002-7143-2500

Deborah Parra-Medina https://orcid.org/0000-0002-5505$524 X$

\section{REFERENCES}

1. Anderson JB. The US-Mexico border: a half century of change. Soc Sci J 2003;40(4):535-554.

2. Mier N, Ory MG, Zhan D, Conkling M, Sharkey JR, Burdine JN. Health-related quality of life among Mexican Americans living in colonias at the Texas-Mexico border. Soc Sci Med 2008;66(8): 1760-1771. 
3. Moore LV, Diez Roux AV, Evenson KR, McGinn AP, Brines SJ. Availability of recreational resources in minority and low socioeconomic status areas. Am J Prev Med 2008;34(1):16-22.

4. Keadle SK, McKinnon R, Graubard BI, Troiano RP. Prevalence and trends in physical activity among older adults in the United States: a comparison across three national surveys. Prev Med 2016;89:37-43.

5. Funk AG. Weight status, physical activity levels, perceived neighborhood health, and healthy community factors among Latinos in greater Cincinnati [dissertation]. Cincinnati: University of Cincinnati; 2016.

6. Floyd MF, Spengler JO, Maddock JE, Gobster PH, Suau LJ. Parkbased physical activity in diverse communities of two U.S. cities. An observational study. Am J Prev Med 2008;34(4):299-305.

7. Kwarteng JL, Schulz AJ, Mentz GB, Zenk SN, Opperman AA. Associations between observed neighborhood characteristics and physical activity: findings from a multiethnic urban community. J Public Health (Oxf) 2014;36(3):358-367.

8. Schulz A, Mentz G, Johnson-Lawrence V, Israel BA, Max P, Zenk $\mathrm{SN}$, et al. Independent and joint associations between multiple measures of the built and social environment and physical activity in a multi-ethnic urban community. J Urban Health 2013;90(5):872-887.

9. Brown SC, Mason CA, Spokane AR, Cruza-Guet MC, Lopez B, Szapocznik J. The relationship of neighborhood climate to perceived social support and mental health in older Hispanic immigrants in Miami, Florida. J Aging Health 2009;21(3):431459.

10. Mellerson J, Landrine H, Hao Y, Corral I, Zhao L, Cooper DL. Residential segregation and exercise among a national sample of Hispanic adults. Health Place 2010;16(3):613-615.

11. Moreno G, Morales LS, Nuñez de Jaimes F, Tseng CH, Isiordia M, Noguera $C$, et al. Neighborhood perceptions and health-related outcomes among Latinos with diabetes from a rural agricultural community. J Community Health 2014;39(6):10771084.

12. Mulvaney-Day NE, Alegría M, Sribney W. Social cohesion, social support, and health among Latinos in the United States. Soc Sci Med 2007;64(2):477-495.

13. Peek MK, Howrey BT, Ternent RS, Ray LA, Ottenbacher KJ. Social support, stressors, and frailty among older Mexican American adults. J Gerontol B Psychol Sci Soc Sci 2012;67(6):755-764.

14. Finch BK, Vega WA. Acculturation stress, social support, and self-rated health among Latinos in California. J Immigr Health 2003;5(3):109-117.
15. Perreira KM, Gotman N, Isasi CR, Arguelles W, Castañeda SF, Daviglus ML, et al. Mental health and exposure to the United States: key correlates from the Hispanic Community Health Study of Latinos. J Nerv Ment Dis 2015;203(9):670-678.

16. Kapulsky L, Tang AM, Forrester JE. Food insecurity, depression, and social support in HIV-infected Hispanic individuals. J Immigr Minor Health 2015;17(2):408-413.

17. Ståhl T, Rütten A, Nutbeam D, Bauman A, Kannas L, Abel T, et al. The importance of the social environment for physically active lifestyle--results from an international study. Soc Sci Med 2001;52(1):1-10.

18. Documet P, Bear TM, Flatt JD, Macia L, Trauth J, Ricci EM. The association of social support and education with breast and cervical cancer screening. Health Educ Behav 2015;42(1):55-64.

19. Luque JS, Opoku S, Ferris DG, Guevara Condorhuaman WS. Social network characteristics and cervical cancer screening among Quechua women in Andean Peru. BMC Public Health 2016;16:181.

20. Lochner KA, Kawachi I, Brennan RT, Buka SL. Social capital and neighborhood mortality rates in Chicago. Soc Sci Med 2003; 56(8):1797-1805.

21. Tucker JM, Welk GJ, Beyler NK. Physical activity in US adults: compliance with the physical activity guidelines for Americans. Am J Prev Med 2011;40(4):454-461.

22. Kozey Keadle S, Lyden K, Staudenmayer J, Hickey A, Viskochil R, Braun $B$, et al. The independent and combined effects of exercise training and reducing sedentary behavior on cardiometabolic risk factors. Appl Physiol Nutr Metab 2014;39(7):770-780.

23. Mailey EL, Rosenkranz SK, Casey K, Swank A. Comparing the effects of two different break strategies on occupational sedentary behavior in a real world setting: a randomized trial. Prev Med Rep 2016;4:423-428.

24. Hawari NS, Al-Shayji I, Wilson J, Gill JM. Frequency of breaks in sedentary time and postprandial metabolic responses. Med Sci Sports Exerc 2016;48(12):2495-2502.

25. Benatti FB, Larsen SA, Kofoed K, Nielsen ST, Harder-Lauridsen NM, Lyngbæk MP, et al. Intermittent standing but not a moderate exercise bout reduces postprandial glycemia. Med Sci Sports Exerc 2017;49(11):2305-2314.

26. Sallis JF, Grossman RM, Pinski RB, Patterson TL, Nader PR. The development of scales to measure social support for diet and exercise behaviors. Prev Med 1987;16(6):825-836.

27. Reis MS, Reis RS, Hallal PC. Validity and reliability of a physical activity social support assessment scale. Rev Saude Publica 2011;45(2):294-301. 
28. Carlson JA, Sallis JF, Conway TL, Saelens BE, Frank LD, Kerr J, et al. Interactions between psychosocial and built environment factors in explaining older adults' physical activity. Prev Med 2012;54(1):68-73.

29. Uchino BN. Social support and health: a review of physiological processes potentially underlying links to disease outcomes. J Behav Med 2006;29(4):377-387.

30. Schulz AJ, Israel BA, Mentz GB, Bernal C, Caver D, DeMajo R, et al. Effectiveness of a walking group intervention to promote physical activity and cardiovascular health in predominantly non-Hispanic black and Hispanic urban neighborhoods: findings from the walk your heart to health intervention. Health Educ Behav 2015;42(3):380-392.

31. Van Dyck D, Cardon G, Deforche B, Sallis JF, Owen N, De Bourdeaudhuij I. Neighborhood SES and walkability are related to physical activity behavior in Belgian adults. Prev Med 2010;50 Suppl 1:S74-S79.

32. Manson JE, Greenland P, LaCroix AZ, Stefanick ML, Mouton CP, Oberman $A$, et al. Walking compared with vigorous exercise for the prevention of cardiovascular events in women. $\mathrm{N}$ Engl J Med 2002;347(10):716-725.
33. Timperio A, Veitch J, Carver A. Safety in numbers: does perceived safety mediate associations between the neighborhood social environment and physical activity among women living in disadvantaged neighborhoods? Prev Med 2015;74:49-54.

34. Van Holle V, Van Cauwenberg J, De Bourdeaudhuij I, Deforche B, Van de Weghe N, Van Dyck D. Interactions between neighborhood social environment and walkability to explain Belgian older adults' physical activity and sedentary time. Int J Environ Res Public Health 2016;13(6):E569.

35. Ferdinand AO, Sen B, Rahurkar S, Engler S, Menachemi N. The relationship between built environments and physical activity: a systematic review. Am J Public Health 2012;102(10):e7e13.

36. Berrigan D, Dodd K, Troiano RP, Reeve BB, Ballard-Barbash R. Physical activity and acculturation among adult Hispanics in the United States. Res Q Exerc Sport 2006;77(2):147-157.

37. González Burchard E, Borrell LN, Choudhry S, Naqvi M, Tsai HJ, Rodriguez-Santana JR, et al. Latino populations: a unique opportunity for the study of race, genetics, and social environment in epidemiological research. Am J Public Health 2005; 95(12):2161-2168. 